\title{
Complementary Roles of Dynamic Contrast-Enhanced MR Imaging and Postcontrast Vessel Wall Imaging in Detecting High-Risk Intracranial Aneurysms
}

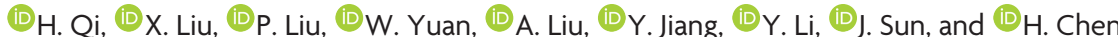

\begin{abstract}
BACKGROUND AND PURPOSE: Individual assessment of the absolute risk of intracranial aneurysm rupture remains challenging. Emerging imaging techniques such as dynamic contrast-enhanced MR imaging and postcontrast vessel wall MR imaging may improve risk estimation by providing new information on aneurysm wall properties. The purpose of this study was to investigate the relationship between aneurysm wall permeability on dynamic contrast-enhanced MR imaging and aneurysm wall enhancement on postcontrast vessel wall MR imaging in unruptured intracranial aneurysms.
\end{abstract}

MATERIALS AND METHODS: Patients with unruptured saccular intracranial aneurysms were imaged with vessel wall MR imaging before and after gadolinium contrast administration. Dynamic contrast-enhanced MR imaging was performed coincident with contrast injection using 3D T1-weighted spoiled gradient-echo imaging. The transfer constant $\left(K^{\text {trans }}\right)$ was measured adjacent to intracranial aneurysm and adjacent to the normal intracranial artery.

RESULTS: Twenty-nine subjects were analyzed (mean age, $53.9 \pm 13.5$ years; $24 \%$ men; PHASES score: median, 8; interquartile range, 4.75-10). $K^{\text {trans }}$ was higher in intracranial aneurysms compared with the normal intracranial artery (median, 0.0110; interquartile range, $0.0060-0.0390$ versus median, 0.0032 ; interquartile range, $0.0018-0.0048 \mathrm{~min}^{-1} ; P<.001$ ), which correlated with intracranial aneurysm size (Spearman $\rho=0.54, P=.002)$ and PHASES score $(\rho=0.40, P=.30)$. Aneurysm wall enhancement, detected in $19(66 \%)$ aneurysms, was associated with intracranial aneurysm size and the PHASES score but not significantly with $K^{\text {trans }}(P=.30)$. Aneurysms of 2 of the 9 patients undergoing conservative treatment ruptured during 1 -year follow-up. Both ruptured aneurysms had increased $K^{\text {trans }}$, whereas only 1 had aneurysm wall enhancement at baseline.

CONCLUSIONS: Dynamic contrast-enhanced MR imaging showed increased $K^{\text {trans }}$ adjacent to intracranial aneurysms, which was independent of aneurysm wall enhancement on postcontrast vessel wall MR imaging. Increased aneurysm wall permeability on dynamic contrast-enhanced MR imaging provides new information that may be useful in intracranial aneurysm risk assessment.

ABBREVIATIONS: $A W E=$ aneurysm wall enhancement; $D C E=$ dynamic contrast-enhanced; $I A=$ intracranial aneurysm; $I Q R=$ interquartile range; $K^{\text {trans }}=$ transfer constant; PHASES = Population, Hypertension, Age, Size, Earlier Subarachnoid Hemorrhage, and Site; WEl = wall enhancement index

ntracranial aneurysm (IA) is a common vascular malformation, affecting approximately $3.2 \%$ of the general population. ${ }^{1,2}$ IA rupture is the most common cause of spontaneous subarach-

Received June 13, 2018; accepted after revision January 2, 2019.

From the Department of Biomedical Engineering (H.Q., X.L., H.C.), Tsinghua University, Beijing, China; Department of Interventional Neuroradiology (P.L., A.L.,Y.J., Y.L.), Beijing Neurosurgical Institute and Beijing Tiantan Hospital, Capital Medical University, Beijing, China; Department of Radiology (W.Y.), Navy Qingdao No. 1 Sanatorium of People's Liberation Army, Qingdao, China; and Department of Radiology (J.S.), University of Washington, Seattle, Washington.

Haikun Qi, Xian Liu and Peng Liu contributed equally to this work.

This work was supported, in part, by the following agencies and organizations: Commission of Beijing Municipal Science and Technology, municipal clinical special application study, the special fund project (No.Z14110000211441); the National Natural Science Foundation (81171078, 81471166, 81771233 and 81441038); Beijing Talents Training Project (Category D) and Beijing Hygiene System High-Level Hygienic Technical Personnel Training Program and the Talents Program of Beijing Tiantan noid hemorrhage, and $80 \%$ of aneurysmal SAHs result in death or permanent neurologic deficits. ${ }^{3}$ Thus, the role of prevention in the clinical management of IAs cannot be overemphasized. Although preemptive treatment with either surgical or endovascular intervention is effective in preventing IA rupture, it also subjects patients to the risk of iatrogenic complications. ${ }^{4,5}$ Thus, individual risk assessment is needed to justify preventive treatment in high-risk patients to

Hospital (Hospital Backbone Program); Capital Health Development Scientific Research Project (2018-2-2041); the American Heart Association (17MCPRP33671077); and the National Center for Advancing Translational Sciences of the National Institutes of Health (UL1 TR002319).

Please address correspondence to Huijun Chen, PhD, Center for Biomedical Imaging Research, Room 109, Tsinghua University, Haidian District, Beijing, 100084, China; e-mail: chenhj_cbir@mail.tsinghua.edu.cn

-*- Indicates open access to non-subscribers at www.ajnr.org

http://dx.doi.org/10.3174/ajnr.A5983 
avoid aneurysmal SAH and predict those who do not need invasive treatment to avoid iatrogenic complications. A number of patientspecific and aneurysm-specific risk factors have been proposed from large prospective cohort studies. ${ }^{4}$ Yet estimating the absolute risk of IA rupture remains difficult.

In most previous studies, ${ }^{4,6}$ IAs were characterized using luminal imaging, which provides little information on patho-

Table 1: Components of the PHASES score ${ }^{14}$

\begin{tabular}{lc}
\hline \multicolumn{1}{c}{ PHASES Aneurysm Risk Score } & Points \\
\hline (P) Population & \\
North American, European (other than Finnish) & 0 \\
Japanese & 3 \\
Finnish & 5 \\
(H) Hypertension & \\
$\quad$ No & 0 \\
Yes & 1 \\
(A) Age & \\
Younger than 70 yr & 0 \\
70 yr or older & 1 \\
(S) Size of aneurysm & \\
$<7.0$ mm & 0 \\
$7.0-9.9$ mm & 3 \\
10.0-19.9 mm & 6 \\
$\geq 20.0$ mm & 10 \\
(E) Earlier SAH from another aneurysm & \\
No & 0 \\
Yes & 1 \\
(S) Site of aneurysm & \\
ICA & \\
MCA & \\
ACA/PcomA/posterior circulation & 2 \\
\hline
\end{tabular}

Note:-ACA indicates anterior cerebral arteries (including the anterior cerebral artery, anterior communicating artery, and pericallosal artery); ICA, internal carotid artery; MCA, middle cerebral artery; PcomA, posterior communicating artery; SAH, subarachnoid hemorrhage.
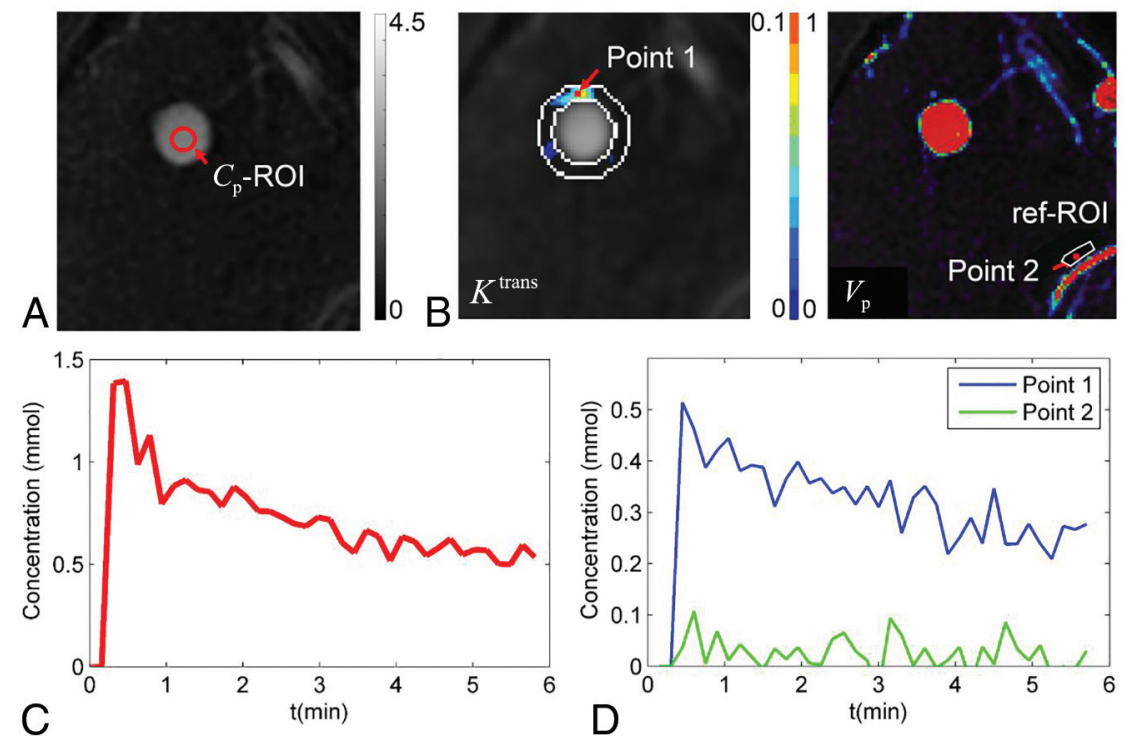

FIG 1. Measuring aneurysm wall permeability by DCE-MR imaging. Signal intensity in the lumen was measured on DCE imaging series (red contour in A) to obtain contrast concentration in plasma at different time points, which gave the arterial input function $C p(t)$ (red curve in $C$ ). The concentration-time curve of tissue $C(t)$ was fitted voxel by voxel using the extended Kety/Tofts model, which generated parametric maps that were overlaid onto DCE images $(B)$. ROls were placed on the slice with highest $K^{\text {trans }}$ to measure $K^{\text {trans }}$ in the region adjacent to intracranial aneurysms (IA-ROI, white contour on $K^{\text {trans }}$ map in $B$ ) and near a normal artery. The concentrationtime curves of points 1 and 2 (red dots in $B$ ) are shown in $D$. ref-ROI indicates reference ROI. physiologic changes or the integrity of the aneurysm wall, which could be more directly associated with IA rupture than IA morphology. Histologic studies of surgically resected IAs showed evidence of aneurysm wall remodeling. ${ }^{7,8}$ Furthermore, during an operation, focal wall thinning of the aneurysm dome was noticeable as translucent regions, which may precede rupture. ${ }^{9}$ Vakil et al ${ }^{10}$ performed dynamic contrast-enhanced (DCE) MR imaging in patients with unruptured IAs and found that the contrast extravasation rate (transfer constant $\left[K^{\text {trans }}\right]$ ) was high in the adjacent region of clinically defined high-risk IAs, indicating a leaky aneurysm wall as a potential marker of high-risk unruptured IAs. On the other hand, with high-resolution vessel wall MR imaging, postcontrast aneurysm wall enhancement (AWE) has been observed, which was also shown to be a marker of high-risk IAs. ${ }^{11,12}$ It is unclear whether increased $K^{\text {trans }}$ on DCE-MR imaging may result from an enhanced aneurysm wall. To better understand the nature of increased $K^{\text {trans }}$ in unruptured IAs, this study sought to investigate the relationship between aneurysm wall permeabil-

\section{MATERIALS AND METHODS} subtraction angiography were invited to participate. Exclusion criteria were contraindications to MR imaging or gadolinium contrast injection. Thirty-two patients ( 24 women; mean age, $54.2 \pm 13.1$ years) with a total of 41 unruptured saccular aneurysms were recruited. Clinical risk assessment was performed by evaluating morphology characteristics on DSA, including IA size, ${ }^{13}$ location (anterior or posterior circulation), and blebs. The Population, Hypertension, Age, Size, Earlier Subarachnoid Hemorrhage, and Site (PHASES) score, ${ }^{14}$ which aggregates multiple clinical risk factors to provide a more accurate estimate of individual risk (Table 1), was calculated. Twenty-three patients received preemptive surgical treatment (clipping: $n=5$; coiling: $n=$ 18). The remaining 9 patients who refused or were not eligible for preemptive surgery were treated conservatively and followed for 1 year.

\section{Imaging Protocol}

Patients were scanned on a 3T wholebody scanner (Achieva TX; Philips Healthcare, Best, the Netherlands) with a 32-channel head coil. First, the target aneurysm was localized by a $3 \mathrm{D}$ time-offlight MRA sequence. For patients with multiple IAs, the referring clinician indicated the target aneurysm for imaging. This was necessary because to ensure 
high temporal and spatial resolution, the DCE-MR imaging used in this study had a limited longitudinal coverage, which made it difficult to simultaneously image multiple IAs that might exist.

The imaging parameters of 3D time-of-flight were the following: $\mathrm{TR} / \mathrm{TE}=25 / 3.5 \mathrm{~ms}$; flip angle $=20^{\circ}$; FOV $=200 \times$ $200 \times 84 \mathrm{~mm}^{3}$; reconstructed voxel size $=0.35 \times 0.35 \times 0.7$ $\mathrm{mm}^{3}$. Before DCE-MR imaging, a 3D black-blood T1weighted volume isotropic turbo spin-echo acquisition sequence $^{15}$ was performed to acquire precontrast vessel wall images with the following parameters: TR/TE $=700 / 30 \mathrm{~ms}$; TSE factor $=49$; FOV $=160 \times 160 \times 54 \mathrm{~mm}^{3}$; voxel size $=0.6 \times$ $0.6 \times 0.6 \mathrm{~mm}^{3} ; 90$ transverse slices. DCE-MR imaging was performed with the imaging slab centered on the target aneurysm. For DCE-MR imaging, precontrast T1 mapping was performed using a 3D variable flip angle sequence with optimized flip angles: $2^{\circ}, 4^{\circ}, 9^{\circ}$, and $25^{\circ} .{ }^{16} \mathrm{~B}_{1}$ mapping was also performed to correct variations of the prescribed flip angle using the actual flip angle method. ${ }^{17}$ Then, DCE images were acquired using a 3D T1-weighted spoiled gradient-echo sequence: TR/ $\mathrm{TE}=3.9 / 2 \mathrm{~ms}$; flip angle $=15^{\circ} ; \mathrm{FOV}=160 \times 160 \mathrm{~mm}^{2} ;$ spatial resolution $=0.8 \times 0.8 \mathrm{~mm}^{2}$; slice thickness $=2 \mathrm{~mm}$; number of slices $=10$; time resolution $=8.8$ seconds. Coincident with the fifth dynamic scan, a bolus of $0.1 \mathrm{mmol} / \mathrm{kg}$ of Gd-DTPA (Magnevist; Bayer HealthCare Pharmaceuticals, Wayne, New Jersey) was injected intravenously at a rate of $1.5 \mathrm{~mL} / \mathrm{s}$, followed by a $20-\mathrm{mL}$ saline flush at the same rate. The total scan time of DCE-MR imaging was about 6 minutes. During the DCE acquisition, a spatial saturation band was positioned proximal to the imaging plane to induce T1 weighting for flowing blood. After DCE-MR imaging, the 3D black-blood T1weighted volume isotropic turbo spin-echo acquisition sequence was repeated for postcontrast vessel wall images with the same imaging parameters as for the precontrast acquisition.

\section{Image Analysis}

The extended Kety/Tofts model ${ }^{18}$ was used to describe the pharmacokinetics of the contrast agent in DCE-MR imaging:

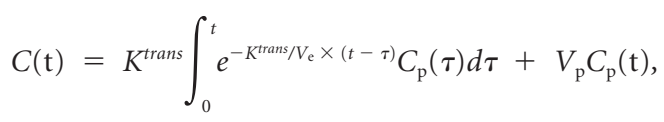

where $K^{\text {trans }}\left(\min ^{-1}\right)$ is the transfer rate of contrast agent from the intravascular space to the extracellular extravascular space; $V_{\mathrm{e}}$ is the extracellular extravascular fractional volume; $V_{\mathrm{p}}$ is the fractional plasma volume; $C(\mathrm{t})$ is the tissue concentration of contrast agent, calculated using signal intensity values in DCE images and precontrast T1 mapping data; and $C_{p}(\mathrm{t})$ is the plasma concentration of contrast agent or arterial input function. ${ }^{19}$ The kinetic model was fitted for each pixel using the least-squares method to generate parametric maps including $K^{\text {trans }}$ and $V_{\mathrm{p}}$ (Fig 1). To measure aneurysm wall permeability, we selected the slice showing the highest $K^{\text {trans }}$ near the aneurysm. An ROI immediately adjacent to the aneurysm was defined by 2 concentric contours: The first was drawn to just enclose the aneurysm, and the second was drawn by expanding the first one 3 pixels away from the aneurysm. Aneurysm wall permeability was measured as mean $K^{\text {trans }}$ within the ROI excluding pixels with blood signal contamination $\left(V_{\mathrm{p}}>0.5\right){ }^{10}$ To measure normal intracranial artery permeability, we placed a reference ROI immediately adjacent to a normal intracranial artery on the same slice.

The pre- and postcontrast 3D T1-weighted volume isotropic turbo spin-echo acquisition images were reformatted into 2-mm slices that matched the DCE-MR images. Blinded to DCE-MR imaging analysis, we evaluated the presence of aneurysm wall enhancement by comparing pre- and postcontrast vessel wall images. ${ }^{20}$ Additionally, the wall-enhancement index $(\text { WEI })^{21}$ was measured to provide a quantitative measurement of aneurysm wall enhancement:

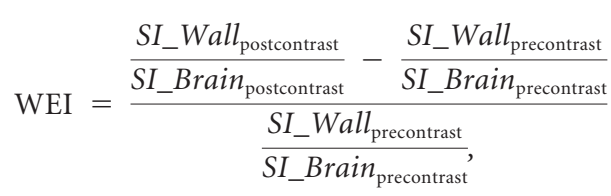

where SI_Brain precontrast and SI_Brain ${ }_{\text {postcontrast }}$ were measured in brain white matter for signal intensity normalization. All imaging measurements were repeated by a second reader to assess interreader reproducibility or reliability.

\section{Data Analysis}

Categoric variables were summarized as count (percentage). The PHASES score was summarized as median (interquartile range, $[\mathrm{IQR}])$. The Kolmogorov-Smirnov test was used to test each continuous variable for whether a normal distribution could be assumed, after which the variable was summarized as mean $\pm \mathrm{SD}$ or median (IQR) as appropriate. The Wilcoxon signed rank test was used to compare $K^{\text {trans }}$ between aneurysms and normal arteries. The Mann-Whitney $U$ test was used to compare $K^{\text {trans }}$ among different aneurysm groups. The Spearman correlation coefficient was used to evaluate the correlation between $K^{\text {trans }}$ and WEI as well as the relationships of $K^{\text {trans }}$ and WEI to age, aneurysm size, and the PHASES score. To assess interreader reproducibility or reliability, we used the intraclass correlation coefficient (2-way model) for $K^{\text {trans }}$ and WEI, while the Cohen $\kappa$ was used for the presence of conspicuous wall enhancement. All statistical analyses were conducted as 2-tailed tests in SPSS (IBM, Armonk, New York). $P<.05$ was defined as statistical significance.

\section{RESULTS}

\section{Patient Demographics}

Three patients were excluded from DCE analysis because of signal contamination from the cavernous sinus after contrast injection. Clinical characteristics of the remaining 29 patients (mean age, $53.9 \pm 13.5$ years; male sex, $24 \%$ ) are summarized in Table 2. Of the 29 IAs, 18 (62\%) were located in the anterior circulation (internal carotid artery, $n=13$; anterior communicating artery, $n=$ 4; middle cerebral artery, $n=1)$, and $11(38 \%)$ were located in the posterior circulation (basilar artery, $n=10$; posterior cerebral artery, $n=1)$. Twenty-three (79\%) patients had a PHASES score of $\geq 4$. 


\section{Aneurysm Wall Permeability by DCE-MR Imaging}

$K^{\text {trans }}$ measured adjacent to an IA was higher than that measured adjacent to a normal intracranial artery (median, 0.0110; IQR, $0.0060-0.0390$ versus median, 0.0032; IQR, 0.0018-0.0048 $\left.\min ^{-1} ; P<.001\right)$. Substantial variations in aneurysm wall permeability were present (Fig 2). Spearman correlation analysis showed that $K^{\text {trans }}$ was moderately correlated with IA size ( $\rho=$ $0.54, P=.002$; Fig 3$)$ and the PHASES score $(\rho=0.40, P=.030)$. Other clinical and imaging characteristics were not significantly associated with $K^{\text {trans }}$.

\section{Aneurysm Wall Enhancement}

Conspicuous wall enhancement was detected in 19 (66\%) aneurysms. IA size and the PHASES score were significantly different between aneurysms with and without AWE (IA size: median, $15.0 \mathrm{~mm}$; IQR, 9.0-22.1 mm versus median, $5.1 \mathrm{~mm}$; IQR, 4.3-8.5; $P<.001$;

Table 2: The demography and aneurysm characteristics of the study sample $(\boldsymbol{N}=29)^{\mathrm{a}}$

\begin{tabular}{|c|c|}
\hline & $\begin{array}{l}\text { No. (\%), Mean, } \\
\text { or Median (IQR) }\end{array}$ \\
\hline Age (yr) & $53.9 \pm 13.5$ \\
\hline Male sex & $7(24 \%)$ \\
\hline Current smoking & $5(17 \%)$ \\
\hline Hypertension & $11(38 \%)$ \\
\hline Diabetes & $2(7 \%)$ \\
\hline \multicolumn{2}{|l|}{ Location } \\
\hline Anterior circulation & $18(62 \%)$ \\
\hline Posterior circulation & $11(38 \%)$ \\
\hline \multicolumn{2}{|l|}{ Size } \\
\hline$<7.0 \mathrm{~mm}$ & $7(24 \%)$ \\
\hline 7.0-9.9 mm & $8(28 \%)$ \\
\hline 10.0-19.9 mm & $7(24 \%)$ \\
\hline$\geq 20.0 \mathrm{~mm}$ & $7(24 \%)$ \\
\hline Blebs & $3(10 \%)$ \\
\hline PHASES score & $8(4.75-10)$ \\
\hline$K^{\operatorname{trans}\left(\min ^{-1}\right)}$ & $0.0107(0.0060-0.0390)$ \\
\hline Aneurysm wall enhancement & $19(66 \%)$ \\
\hline
\end{tabular}

a See Table 1 for details of the PHASES score.
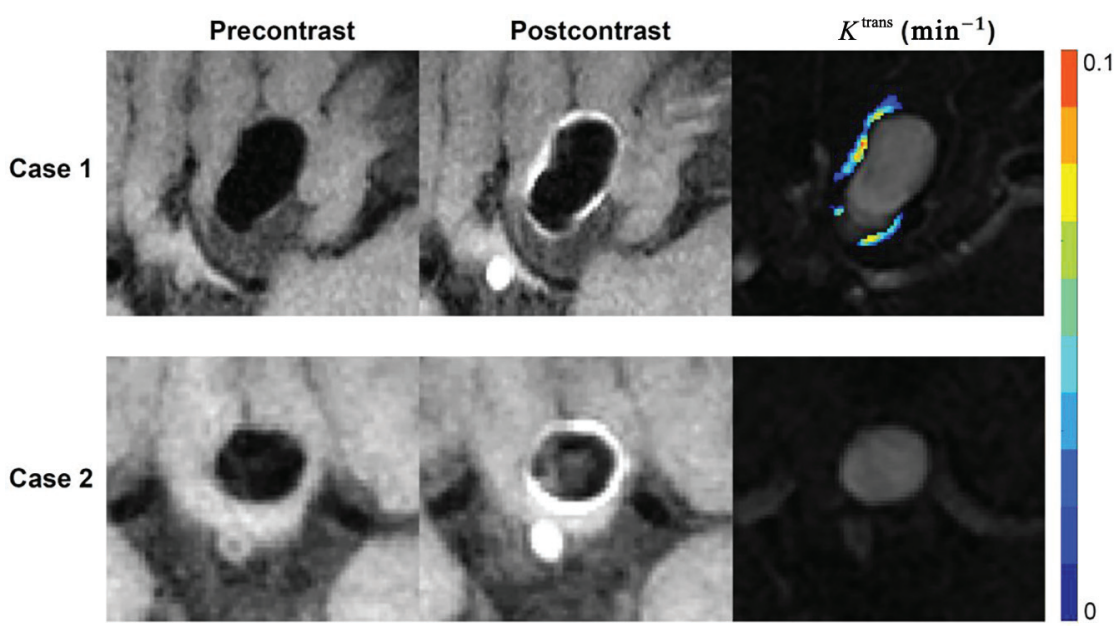

FIG 2. Enhanced aneurysm wall with and without increased permeability. Case 1: A 51-year-old woman who presented with a 19.5-mm aneurysm in the internal carotid artery. Pre- and postcontrast vessel wall images show conspicuous wall enhancement. DCE-MR imaging shows increased wall permeability $\left(K^{\text {trans }}=0.0346 \mathrm{~min}^{-1}\right)$. Case 2: A 61-year-old woman who presented with a $9-\mathrm{mm}$ aneurysm in the anterior communicating artery. Pre- and postcontrast vessel wall imaging shows conspicuous wall enhancement, but DCE-MR imaging did not show increased wall permeability $\left(K^{\text {trans }}=0.0096 \mathrm{~min}^{-1}\right)$.
PHASES score: median, 10; IQR, 8-10.75 versus median 3.5; IQR, $2-5 ; P<.001)$. As a quantitative measure of AWE, the median WEIs were 4.0 (IQR, 2.4-6.7) and 1.4 (IQR, 1.1-1.5) in aneurysms with and without conspicuous wall enhancement, respectively; $P<.001$. By contrast, $K^{\text {trans }}$ was not significantly associated with AWE (median, 0.0157; IQR, 0.0065-0.0421 versus median, 0.0089; IQR, $0.0059-0.0338 \mathrm{~min}^{-1} ; P=.30$; Fig 4$)$. The scatterplot in Fig 4 shows a poor concordance between $K^{\text {trans }}$ and the WEI in individual patients despite a statistically significant correlation coefficient in the overall sample $(\rho=0.39, P=.040)$.

\section{Follow-Up Findings}

During the 1-year follow-up, 2 of the 9 patients undergoing conservative treatment had aneurysmal SAH at 1 month and 5 months, respectively. Figure 5 shows the inhomogeneous $K^{\text {trans }}$ maps and vessel wall images of the 2 aneurysms that ruptured within a year. Notably, both ruptured aneurysms had high $K^{\text {trans }}$ in the region adjacent to the aneurysm, whereas only 1 had obvious wall enhancement on baseline MR imaging. In fact, the 2 ruptured aneurysms had the highest and second highest baseline $K^{\text {trans }}$ among the 9 aneurysms that were followed. Baseline $K^{\text {trans }}$ was significantly higher in the ruptured aneurysms than in the other aneurysms $(P=.040)$, whereas IA size, the PHASES score, and WEI were not significantly different between the 2 groups $(P=.56,>.99$, and .38 , respectively $)$.

\section{Repeat Measurements}

All MR imaging measurements were repeated by an independent reader to evaluate interreader reproducibility or reliability. The intraclass correlation coefficients were 0.991 (95\% CI, $0.981-$ $0.996)$ and 0.74 (95\% CI, 0.51-0.87) for $K^{\text {trans }}$ and WEI, respectively. The Cohen $\kappa$ for the presence of conspicuous wall enhancement was 0.79 (95\% CI, 0.56-1.00).

\section{DISCUSSION}

DCE-MR imaging and high-resolution vessel wall MR imaging are emerging techniques that may provide information about aneurysm wall properties relevant to IA rupture. ${ }^{10,20,21}$ However, the nature of the imaging findings by these techniques remains elusive given the difficulties with histopathologic validation. To our knowledge, this is the first study to evaluate the relationship between increased $K^{\text {trans }}$ on DCE-MR imaging and AWE on vessel wall MR imaging. Our observations support increased $K^{\text {trans }}$ as a distinctive finding in high-risk IAs rather than explaining it by the coexisting AWE. Therefore, increased $K^{\text {trans }}$ likely represents contrast leakage from the aneurysm into the adjacent region captured by timeresolved imaging. Measuring aneurysm wall permeability has the potential to allow more precise risk assessment of IA rupture.

DCE-MR imaging collects a time-se- 

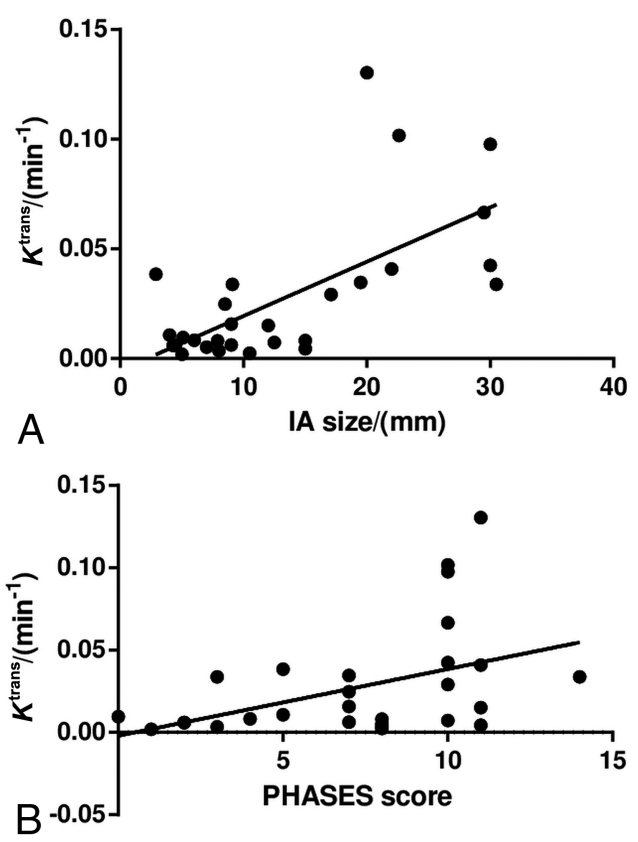

FIG 3. Associations between aneurysm wall permeability and size $(A)$ and the PHASES score $(B)$. Spearman correlation analysis shows that $K^{\text {trans }}$ is positively correlated with aneurysm size $(\rho=0.54, P=.002)$ and the PHASES score $(\rho=0.40, P=.030)$.
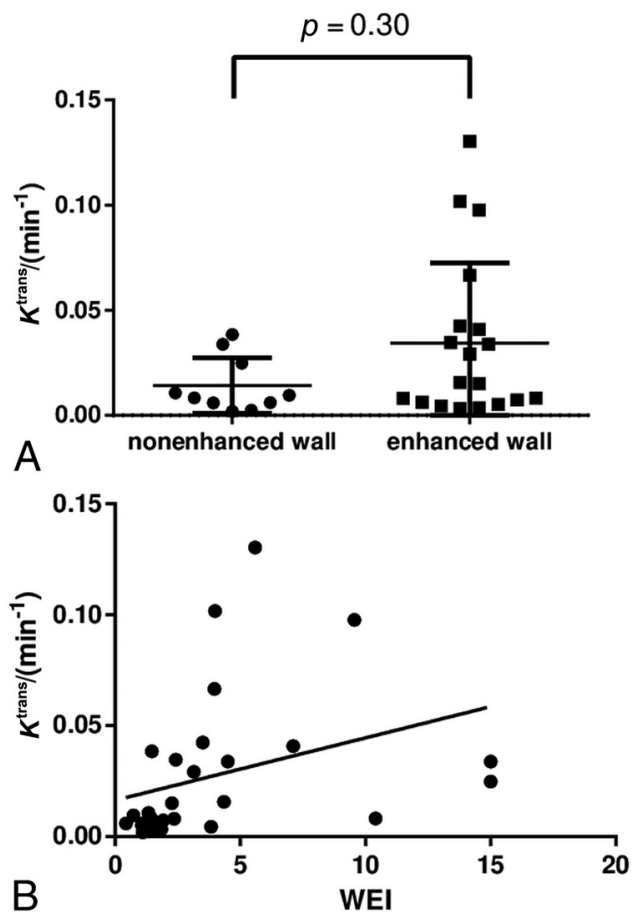

FIG 4. Association between $K^{\text {trans }}$ and aneurysm wall enhancement. $K^{\text {trans }}$ shows no statistically significant association with the presence of aneurysm wall enhancement $(A)$ and a poor concordance with the wall enhancement index $(B)$.

ries of contrast-enhanced images on intravenous injection of gadolinium contrast. Data are then subject to pharmacokinetic modeling to estimate the contrast extravasation rate $\left(K^{\text {trans }}\right)$, which reflects vessel permeability. In a recent study, Vakil et al ${ }^{10}$ applied DCE-MR imaging to 27 unruptured IAs and noted that some aneurysms appeared to be "leaky," supported by increased $K^{\text {trans }}$ in the region adjacent to aneurysm. In vivo detection of leaky aneurysm walls may allow us to capture an asymptomatic, precursor stage of IA rupture, with important implications for the prevention of aneurysmal SAH. However, the relationship between increased $K^{\text {trans }}$ on DCE-MR imaging and AWE on vessel wall MR imaging needs to be clarified. First, high $K^{\text {trans }}$ can result from AWE in the presence of partial volume effects and/or aneurysm wall motion. Second, assessing AWE is easier to do clinically than measuring $K^{\text {trans }}$. Thus, $K^{\text {trans }}$ must provide pathologic information different from that provided by AWE to justify its clinical utility.

Consistent with Vakil et al, ${ }^{10}$ we also observed that $K^{\text {trans }}$ measured adjacent to the IA was higher (leakier) than that measured adjacent to the normal intracranial artery. Of note, $66 \%$ of the studied aneurysms had conspicuous wall enhancement (AWE), with a median WEI of 4.0 (IQR, 2.4-6.7) compared with 1.4 (IQR, 1.1-1.5) in those without AWE. Nonetheless, further analyses indicated that high $K^{\text {trans }}$ adjacent to an IA was unlikely due to AWE. First, about half of the aneurysms with AWE did not have increased $K^{\text {trans }}$, while aneurysms without AWE could have increased $K^{\text {trans }}$. Second, although $K^{\text {trans }}$ had a statistically significant association with WEI, the correlation was weak ( $\rho=0.39$, $P=.040)$. In fact, the correlation of $K^{\text {trans }}$ with IA size appeared stronger $(\rho=0.54, P=.002)$, suggesting that $K^{\text {trans }}$ and WEI are unlikely to represent different imaging measurements of the same pathology. Most interesting, IA rupture was seen during follow-up in the 2 aneurysms with the highest baseline $K^{\text {trans }}$, but only 1 had AWE. Overall, our data support increased $K^{\text {trans }}$, reflecting a pathophysiologic phenomenon that is different from AWE.

The exact pathophysiology of increased IA wall permeability remains speculative. Nonetheless, histopathologic findings, intraoperative observations, and patient symptoms appear to support the existence of focally present, thin, and leaky wall regions that may predispose to rupture. Kataoka et $\mathrm{al}^{8}$ compared 44 ruptured and 27 unruptured IA specimens and found that ruptured aneurysms more often had disrupted endothelial linings and hyalinelike wall structures that may result from inflammatory cell infiltration. Kadasi et al ${ }^{22}$ described translucent wall regions under an intraoperative microscope that represented focal wall thinning and correlated with the distribution of low wall shear stress. Furthermore, many patients with aneurysmal SAH recalled that they had a warning headache before $\mathrm{SAH}$, which is thought to indicate a minor leak. ${ }^{23,24}$ Alternatively, a vasa vasorum, which has been noted in the histologic examination of aneurysm walls, ${ }^{25}$ may be the source of contrast medium leakage into the surrounding CSF. In atherosclerotic plaque, a vasa vasorum has been shown to be leaky on DCE-MR imaging, particularly under inflammatory conditions. ${ }^{26}$ Radiologic-pathologic correlation studies are critically needed to elucidate the underlying pathophysiologic mechanism of increased IA wall permeability on DCE-MR imaging.

$K^{\text {trans }}$ measured in this study was lower than that reported by Vakil et al. ${ }^{10}$ This finding can be explained by the different methods that the 2 studies used to measure mean $K^{\text {trans }}$ on parametric maps. While the previous study used a single contour to define an ROI ( $\geq 10$ pixels) that was placed adjacent to aneurysm wall to measure the " $K^{\text {trans }}$ hotspot," the present study used 2 concentric 


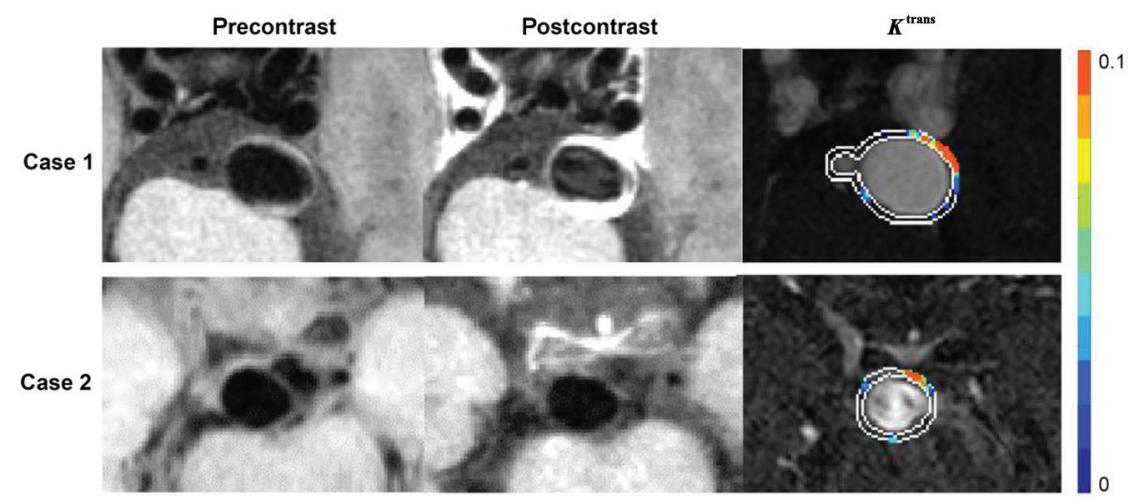

FIG 5. Baseline MR images of the 2 aneurysms that ruptured during follow-up. Case 1: A 36-yearold man has a 17.1-mm aneurysm in the basilar artery. The patient was concerned about surgical risk and received conservative treatment. Subarachnoid hemorrhage occurred 5 months after the baseline scan (no aneurysm wall enhancement; $K^{\text {trans }}=0.0449 \mathrm{~min}^{-1}$ ). Case 2: A 68-year-old man had an $8.5-\mathrm{mm}$ aneurysm in the basilar artery. The patient was concerned about surgical risk and received conservative treatment. Subarachnoid hemorrhage occurred 1 month after baseline scan (no aneurysm wall enhancement; $K^{\text {trans }}=0.0524 \mathrm{~min}^{-1}$ ).

contours to define an ROI that enclosed the entire circumferential region. In the former, ROIs of different sizes may generate different $K^{\text {trans }}$ measurements from the same $K^{\text {trans }}$ map. The ROI in the latter method is more objectively defined, which may facilitate comparison of $K^{\text {trans }}$ measurements among subjects. However, it is possible that the risk of IA rupture could be determined by a leaky wall segment that is focally present. Therefore, both methods may have limitations. Similar results were found (data not shown) when image review was redone using the single-contour method. Nonetheless, a standardized method to obtain segmental wall permeability (eg, fixed size ROI) of an IA may be worth exploring in the future.

The International Study of Unruptured Intracranial Aneurysms reported that the risk of IA rupture increased with increased IA size. ${ }^{4}$ Currently, IA size is arguably the most important factor clinically to determine the risk of IA rupture. However, given the much higher prevalence of small IAs than large ones, aneurysmal SAH most frequently results from small IAs. ${ }^{27,28}$ Recent efforts have attempted to aggregate multiple risk factors into clinical risk scores, such as the PHASES score. ${ }^{14}$ External data indicated that the PHASES score may only have moderate sensitivity (75\%) and specificity (64\%) in detecting high-risk IAs, ${ }^{29}$ which would translate into $25 \%$ of high-risk IAs left untreated and $36 \%$ of treated IAs being low-risk ones. In this context, the moderate correlations of $K^{\text {trans }}$ with IA size and the PHASES score add to the expectation that assessing aneurysm wall permeability may eventually allow clinicians to better identify individuals at risk of IA rupture than current risk markers. However, this expectation is beyond the scope of the present study and will await future largescale investigations as more centers accrue experience with the technique.

Several important limitations of this study are worth mentioning. First, this is a proof-of-concept study with a relatively small sample size. Despite the promising results, the robustness of DCE-MR imaging in measuring IA permeability remains to be explored more extensively in future research to understand factors that may compromise the accuracy of the $K^{\text {trans }}$ measurement, especially if $K^{\text {trans }}$ is studied for potential use in the man- agement of individual patients. To eliminate any influence from aneurysm wall motion, cardiac triggering using electrocardiography or a peripheral pulse unit may be used in data acquisition. However, because cardiac gating will reduce the imaging efficiency and increase the scan time, different $k$-space trajectories and advanced reconstruction algorithms may be required to accelerate DCE-MR imaging. ${ }^{30}$ Second, the DCE-MR imaging sequence used in this study provides limited longitudinal coverage $(20 \mathrm{~mm})$. It is impossible to cover multiple aneurysms in 1 DCE-MR imaging scan. Thus, a priori selection of the target aneurysm is necessary in patients with multiple aneurysms. Furthermore, image analysis for certain ICA aneurysms can be difficult due to strong enhancement of the cavernous sinus. Third, associations of imaging findings with intraoperative observations and/or histologic findings were not performed, which could have provided further insights into the nature of increased $K^{\text {trans }}$ on DCE-MR imaging. Last, the test-retest reproducibility of $K^{\text {trans }}$ remains to be studied. The interreader reproducibility of $K^{\text {trans }}$ was found to be excellent; the outcome was not surprising because most steps in DCE-MR imaging analysis were automatic. However, it will be critical to evaluate its test-retest reproducibility.

\section{CONCLUSIONS}

In our series of unruptured IAs referred to the neurosurgery clinics, DCE-MR imaging showed increased $K^{\text {trans }}$ in the adjacent region of the IA compared with the normal intracranial artery. Such increases in $K^{\text {trans }}$ were not explained by AWE on vessel wall MR imaging, though both were associated with large IA size. Therefore, increased $K^{\text {trans }}$ likely represents a real phenomenon of increased aneurysmal wall permeability, which provides different information from AWE. Future studies are warranted to evaluate its prognostic value independent of clinical risk assessment.

Disclosures: Xian Liu-RELATED: Grant: National Natural Science Foundation of China, the American Heart Association, Comments: This work was supported, in part, by the following agencies and organizations: Commission of Beijing Municipal Science and Technology, municipal clinical special application study, the special fund project (No. Z14110000211441); the National Natural Science Foundation (81171078, 81471166, 81771233, and 81441038); Beijing Talents Training Project (Category D) and Beijing Hygiene System High-Level Hygienic Technical Personnel Training Program and the Talents Program of Beijing Tiantan Hospital (Hospital Backbone Program); Capital Health Development Scientific Research Project (2018-2-2041); the American Heart Association (17MCPRP33671077); the National Center for Advancing Translational Sciences of the National Institutes of Health (UL1 TR002319).* Jie Sun-UNRELATED: Grants/Grants Pending: American Heart Association.* Huijun Chen-RELATED: Grant: National Natural Science Foundation of China, Comments: National Natural Science Foundation of China funding 81571667 and 81371540 .* *Money paid to the institution.

\section{REFERENCES}

1. Thompson BG, Brown RD Jr, Amin-Hanjani S, et al; American Heart Association Stroke Council, Council on Cardiovascular and Stroke Nursing, and Council on Epidemiology and Prevention, American Heart Association, American Stroke Association. Guidelines for the Manage-

AJNR Am J Neuroradiol 40:490-96 Mar 2019 www.ajnr.org 
ment of Patients with Unruptured Intracranial Aneurysms: a Guideline for Healthcare Professionals from the American Heart Association/American Stroke Association. Stroke 2015;46:2368-400 CrossRef Medline

2. Vlak MH, Algra A, Brandenburg R, et al. Prevalence of unruptured intracranial aneurysms, with emphasis on sex, age, comorbidity, country, and time period: a systematic review and meta-analysis. Lancet Neurol 2011;10:626-36 CrossRef Medline

3. van Gijn J, Kerr RS, Rinkel GJE. Subarachnoid haemorrhage. Lancet 2007;369:306-18 CrossRef Medline

4. Wiebers DO, Whisnant JP, Huston J 3rd, et al; International Study of Unruptured Intracranial Aneurysms Investigators. Unruptured intracranial aneurysms: natural history, clinical outcome, and risks of surgical and endovascular treatment. Lancet 2003;362:103-10 CrossRef Medline

5. Raaymakers TW, Rinkel GJ, Limburg M, et al. Mortality and morbidity of surgery for unruptured intracranial aneurysms: a metaanalysis. Stroke 1998;29:1531-38 CrossRef Medline

6. Morita A, Kirino T, Hashi K, et al; UCAS Japan Investigators. The natural course of unruptured cerebral aneurysms in a Japanese cohort. $N$ Engl J Med. 2012;366:2474-82 CrossRef Medline

7. Frösen J, Piippo A, Paetau A, et al. Remodeling of saccular cerebral artery aneurysm wall is associated with rupture: histological analysis of 24 unruptured and 42 ruptured cases. Stroke 2004;35:2287-93 CrossRef Medline

8. Kataoka K, Taneda M, Asai T, et al. Structural fragility and inflammatory response of ruptured cerebral aneurysms: a comparative study between ruptured and unruptured cerebral aneurysms. Stroke 1999;30:1396-401 CrossRef Medline

9. Kadasi LM, Dent WC, Malek AM. Colocalization of thin-walled dome regions with low hemodynamic wall shear stress in unruptured cerebral aneurysms. J Neurosurg 2013;119:172-79 CrossRef Medline

10. Vakil P, Ansari SA, Cantrell CG, et al. Quantifying intracranial aneurysm wall permeability for risk assessment using dynamic contrast-enhanced MRI: a pilot study. AJNR Am J Neuroradiol 2015;36: 953-59 CrossRef Medline

11. Matouk CC, Mandell DM, Günel M, et al. Vessel wall magnetic resonance imaging identifies the site of rupture in patients with multiple intracranial aneurysms: proof of principle. Neurosurgery 2013; 72:492-96; discussion 496 CrossRef Medline

12. Nagahata S, Nagahata M, Obara $M$, et al. Wall enhancement of the intracranial aneurysms revealed by magnetic resonance vessel wall imaging using three-dimensional turbo spin-echo sequence with motion-sensitized driven-equilibrium: a sign of ruptured aneurysm? Clin Neuroradiol 2016;26:277-83 CrossRef Medline

13. Backes D, Vergouwen MDI, Velthuis BK, et al. Difference in aneurysm characteristics between ruptured and unruptured aneurysms in patients with multiple intracranial aneurysms. Stroke 2014;45: 1299-303 CrossRef Medline

14. Greving JP, Wermer MJ, Brown RD Jr, et al. Development of the PHASES score for prediction of risk of rupture of intracranial aneurysms: a pooled analysis of six prospective cohort studies. Lancet Neurol 2014;13:59-66 CrossRef Medline

15. Qiao Y, Steinman DA, Qin Q, et al. Intracranial arterial wall imaging using three-dimensional high isotropic resolution black blood MRI at 3.0 Tesla. J Magn Reson Imaging 2011;34:22-30 CrossRef Medline

16. Cheng HL, Wright GA. Rapid high-resolution T(1) mapping by variable flip angles: accurate and precise measurements in the presence of radiofrequency field inhomogeneity. Magn Reson Med 2006; 55:566-74 CrossRef Medline

17. Yarnykh VL. Actual flip-angle imaging in the pulsed steady state: a method for rapid three-dimensional mapping of the transmitted radiofrequency field. Magn Reson Med 2007;57:192-200 CrossRef Medline

18. Tofts PS, Brix G, Buckley DL, et al. Estimating kinetic parameters from dynamic contrast-enhanced T1-weighted MRI of a diffusable tracer: standardized quantities and symbols. J Magn Reson Imaging 1999;10;223-32 CrossRef Medline

19. Yang C, Karczmar GS, Medved M, et al. Reproducibility assessment of a multiple reference tissue method for quantitative dynamic contrast enhanced-MRI analysis. Magn Reson Med 2009;61:851-59 CrossRef Medline

20. Liu P, Qi H, Liu A, et al. Relationship between aneurysm wall en hancement and conventional risk factors in patients with unruptured intracranial aneurysms: a black-blood MRI study. Interv Neuroradiol 2016;22:501-05 CrossRef Medline

21. Omodaka S, Endo H, Niizuma K, et al. Quantitative assessment of circumferential enhancement along the wall of cerebral aneurysms using MR imaging. AJNR Am J Neuroradiol 2016;37:1262-66 CrossRef Medline

22. Kadasi LM, Dent WC, Malek AM. Cerebral aneurysm wall thickness analysis using intraoperative microscopy: effect of size and gender on thin translucent regions. J Neurointerv Surg 2013;5:201-06 CrossRef Medline

23. Juvela S. Minor leak before rupture of an intracranial aneurysm and subarachnoid hemorrhage of unknown etiology. Neurosurgery 1992;30:7-11 CrossRef Medline

24. Oda S, Shimoda M, Hirayama A, et al. Neuroradiologic diagnosis of minor leak prior to major SAH: diagnosis by T1-FLAIR mismatch. AJNR Am J Neuroradiol 2015;36:1616-22 CrossRef Medline

25. Larsen N, von der Brelie C, Trick D, et al. Vessel wall enhancement in unruptured intracranial aneurysms: an indicator for higher risk of rupture? High-resolution MR imaging and correlated histologic findings. AJNR Am J Neuroradiol 2018;39:1617-21 CrossRef Medline

26. Wang J, Chen H, Sun J, et al. Dynamic contrast-enhanced MR imaging of carotid vasa vasorum in relation to coronary and cerebrovascular events. Atherosclerosis 2017;263:420-26 CrossRef Medline

27. Forget TRJ, Benitez R, Veznedaroglu E, et al. A review of size and location of ruptured intracranial aneurysms. Neurosurgery 2001;49. 1322-25; discussion 1325-26 CrossRef Medline

28. Weir B, Disney L, Karrison T. Sizes of ruptured and unruptured aneurysms in relation to their sites and the ages of patients. $J \mathrm{Neu}$ rosurg 2002;96:64-70 Medline

29. Bijlenga P, Gondar R, Schilling S, et al. PHASES score for the management of intracranial aneurysm: a cross-sectional populationbased retrospective study. Stroke 2017;48:2105-12 CrossRef Medline

30. Feng L, Grimm R, Block KT, et al. Golden-angle radial sparse parallel MRI: combination of compressed sensing, parallel imaging, and golden-angle radial sampling for fast and flexible dynamic volumetric MRI. Magn Reson Med 2014;72:707-17 CrossRef Medline 